\title{
Mesangial Function in Ureteral Obstruction in the Rat
}

\author{
BLOCKADE OF THE EFFERENT LIMB
}

\author{
Leopoldo Raij, William F. Keane, Hartmuth Osswald, and Alfred Michael, \\ Departments of Medicine and Pediatrics, University of Minnesota Medical \\ School, Minneapolis, Minnesota 55455
}

\begin{abstract}
A в S T R A C T The kinetics for mesangial uptake and transport of radiolabeled aggregated human immunoglobulin (Ig)G (AHIgG ${ }^{125}$ I) deviated markedly from normal in male Sprague-Dawley rats with ureteral obstruction. Four experimental groups, each containing 25 rats, were used: $(a)$ bilateral ureteral ligation (BUL) with release of one ureter $24 \mathrm{~h}$ later; (b) unilateral ureteral ligation with release $24 \mathrm{~h}$ later [UUL(R)]; (c) unilateral ureteral ligation without release (unreleased) [UUL(U)]; (d) uremia-control, which consisted of rats with ligated left ureter and a severed right ureter. A similar number of sham-operated rats served as control for each group. AHIgG ${ }^{125}$ ( $45 \mathrm{mg} / 100 \mathrm{~g}$ body wt) was given intravenously $1 \mathrm{~h}$ after release of the ureteral obstruction ( $25 \mathrm{~h}$ after ureteral obstruction or sham surgery). Groups of five control and five experimental animals were sacrificed at $2,4,8,16$, and $24 \mathrm{~h}$ after injection.
\end{abstract}

At all time intervals, concentrations of $\mathrm{AHIgG}^{125} \mathrm{I}$ in isolated glomeruli from control animals were similar to values obtained from nonobstructed kidneys of $\mathrm{UUL}(\mathrm{U})$ and $\mathrm{UUL}(\mathrm{R})$ rats: a linear decrease in concentration over a period of $24 \mathrm{~h}$ was observed when the logarithm of glomerular $\mathrm{AHIgG}^{125}$ I concentration was plotted against time. Aberrations in the kinetics were apparent in obstructed kidneys but not in liver, spleen, or blood concentrations of AHIgG ${ }^{125} \mathrm{I}$ : (a) At $2 \mathrm{~h}$ in all obstructed kidneys, glomerular concentration of AHIgG $^{125}$ I was markedly reduced. (b) In BUL (released or unreleased), glomerular concentrations of $\mathrm{AHIgG}^{125} \mathrm{I}$

This study was presented in part at the National Meeting of the American Society for Clinical Investigation, San Francisco, Calif., May 1978, and at the conference on "Immunological mechanisms in renal disease," sponsored by the National Institutes of Health, Bethesda, Md., October 1978.

Dr. Osswald's current address is Abteilung Pharmakologie, an der Rhein-Westf. Techn., Hochschule Aachen, West Germany. Address reprint requests to Dr. Raij.

Received for publication 21 November 1978 and in revised form 9 July 1979. from 4 to $16 \mathrm{~h}$ were $\cong 10$-fold those in $\mathrm{UUL}(\mathrm{U})$ or $\mathrm{UUL}(\mathrm{R})$ kidneys. (c) The significant decline in glomerular concentration between 4 and $16 \mathrm{~h}$ in control and nonobstructed kidneys was not observed in UUL(R), UUL(U), or BUL (released or unreleased) kidneys; in all obstructed kidneys, a plateau in glomerular concentrations of AHIgG $^{125}$ I was observed between 4 and $16 \mathrm{~h} .(d)$ After $16 \mathrm{~h}$ at a time when the blood level of $\mathrm{AHIgG}^{125} \mathrm{I}$ had decreased to $3 \%$ of initial values, there was progressive fall in glomerular $\mathrm{AHIgG}^{125}$ I. Similar results were obtained in the uremia-control group in rats, which indicated that uremia per se had no measurable effect on mesangial kinetics. These studies demonstrate that ureteral occlusion induces alterations in mesangial uptake (afferent limb) and egress (efferent limb) of macromolecules. Particularly evident is the "blockade" of the efferent limb which is demonstrable at high blood levels of $\mathrm{AHIgG}^{125}$. These alterations in the transit of macromolecules through the mesangium may be mediated in part by the hemodynamic changes that accompany ureteral obstruction.

\section{INTRODUCTION}

The glomerular mesangium occupies an intercapillary position and consists of mesangial cells and an amorphous fibrillar material, the mesangial matrix (1). Anatomically, it is separated from the capillary lumen by the endothelium and limited by the mesangial reflexion of the glomerular basement membrane (2). The contiguity with the lacis cells of the juxtaglomerular $(\mathrm{JG})^{1}$ zone and the presence of actomyosin suggest that it may play a role in the regulation of the glomerular

${ }^{1}$ Abbreviations and nomenclature used in this paper: AHIgG ${ }^{125}$ I, radiolabeled aggregated human IgG; BUL, bilateral ureteral ligation; BUN, blood urea nitrogen; GFR, glomerular filtration rate; JG, juxtaglomerular; $\mathrm{P}_{\mathrm{GC}}$, glomerular capillary pressure; RBF, renal blood flow; SNGFR, single nephron GFR; UUL(R), unilateral ureteral ligation with left ureter released; $U U L(U)$, unilateral ureteral ligation with left ureter unreleased. 
blood flow $(3,4)$. In addition, morphological studies with a variety of probes have demonstrated movement of macromolecules into the mesangium by way of the endothelial fenestra and the mesangial endothelial interface (5-8). This mesangial uptake (afferent limb) is dependent in part upon type, size, and blood level of the macromolecule (8). The mechanisms controlling the clearing of macromolecules (efferent limb) are incompletely understood, but depend upon phagocytosis, passage by way of the glomerular stalk to the JG region, and possibly regurgitation into the glomerular circulation (8).

Whether the hemodynamic determinants of glomerular filtration modulate the mesangial traffic of macromolecules has not been defined. Unilateral and bilateral ureteral ligation are known to induce alterations in these determinants, particularly renal blood flow (RBF), glomerular capillary pressure $\left(\mathrm{P}_{\mathrm{GC}}\right)$, and tubular pressure (9-14). Ureteral obstruction is also accompanied by changes in the renal interstitium and renal lymph flow $(15,16)$. We have studied mesangial uptake and disappearance of radiolabeled aggregated human immunoglobulin (Ig)G (AHIgG $\left.{ }^{125} \mathrm{I}\right)$, a polydispersed population of macromolecules with characteristics similar to those of antigen-antibody complexes, in normal rats and in rats with unilateral and bilateral ureteral ligation of 24-h duration. In the experiments reported here, we have shown a marked alteration in mesangial uptake and egress of macromolecules after ureteral obstruction of 24 -h duration. Specifically, ureteral occlusion induces a "blockade" in the transport of AHIgG ${ }^{125}$ I out of the mesangium (efferent limb) which is demonstrable at high blood levels of $\mathrm{AHIgG}^{125} \mathrm{I}$.

\section{METHODS}

\section{Preparation of $\mathrm{AHIgG}{ }^{125} \mathrm{I}$}

Human immune serum globulin (E. R. Squibb and Sons, New Brunswick, N. J.) was labeled with ${ }^{125}$ (New England Nuclear, Boston, Mass.) by the chloramine-T method of McConahey and Dixon (17). The efficiency of labeling was $\cong 55 \%$, and the quantity of free ${ }^{125} \mathrm{I}$ in the final product was $<3 \%$. From this labeled protein, $\mathrm{AHIgG}^{125}$ I was prepared by heat aggregation according to the methods of Christian (18) and Ishizaka and Ishizaka (19) as modified by Mauer et al. $(6,7)$.

The final concentration of $\mathrm{AHIgG}^{125}$ I was adjusted to contain $60 \mathrm{mg}$ of protein $/ \mathrm{ml}$; merthiolate was added to a final concentration of $1: 10,000$. Free ${ }^{125} \mathrm{I}$ in this preparation was $<1 \%$, and the specific activity of the solution was $0.1 \mathrm{mCi} / \mathrm{mg}$ of protein. The AHIgG ${ }^{125}$ I was stored at room temperature and used within a week of its preparation. Immediately before the injection of $\mathrm{AHIgG}^{125}$ I to animals, the solution was centrifuged at $5,000 \mathrm{~g}$ to remove insoluble aggregates.

\section{Experimental design}

Male Sprague-Dawley rats (Bio-Lab Corp., St. Paul, Minn.) weighing $190-210 \mathrm{~g}$ were used in all experiments. The animals were housed in cages and allowed free access to water and standard Purina rat chow (Ralston Purina Co., St. Louis, Mo.) up to the time of the experiment.

Experimental and control groups (Fig. 1). Four experimental groups, each consisting of 25 animals, were used: Group 1, bilateral ureteral ligation (BUL) in which both ureters were obstructed and $24 \mathrm{~h}$ later the left ureter was released. Group 2, unilateral ureteral ligation in which the left ureter was obstructed and $24 \mathrm{~h}$ later was released [UUL(R)]. Group 3, unilateral ligation in which the left ureter was obstructed for 24 h but not released [UUL(U)]. Group 4, "uremiacontrol," effect of uremia on the mesangial kinetics of AHIgG ${ }^{125}$ I in obstructed and unobstructed kidneys; after overnight dehydration, the right ureter was severed and allowed to drain into the peritoneal cavity while the left ureter was ligated and released $24 \mathrm{~h}$ later. For each of the four experimental

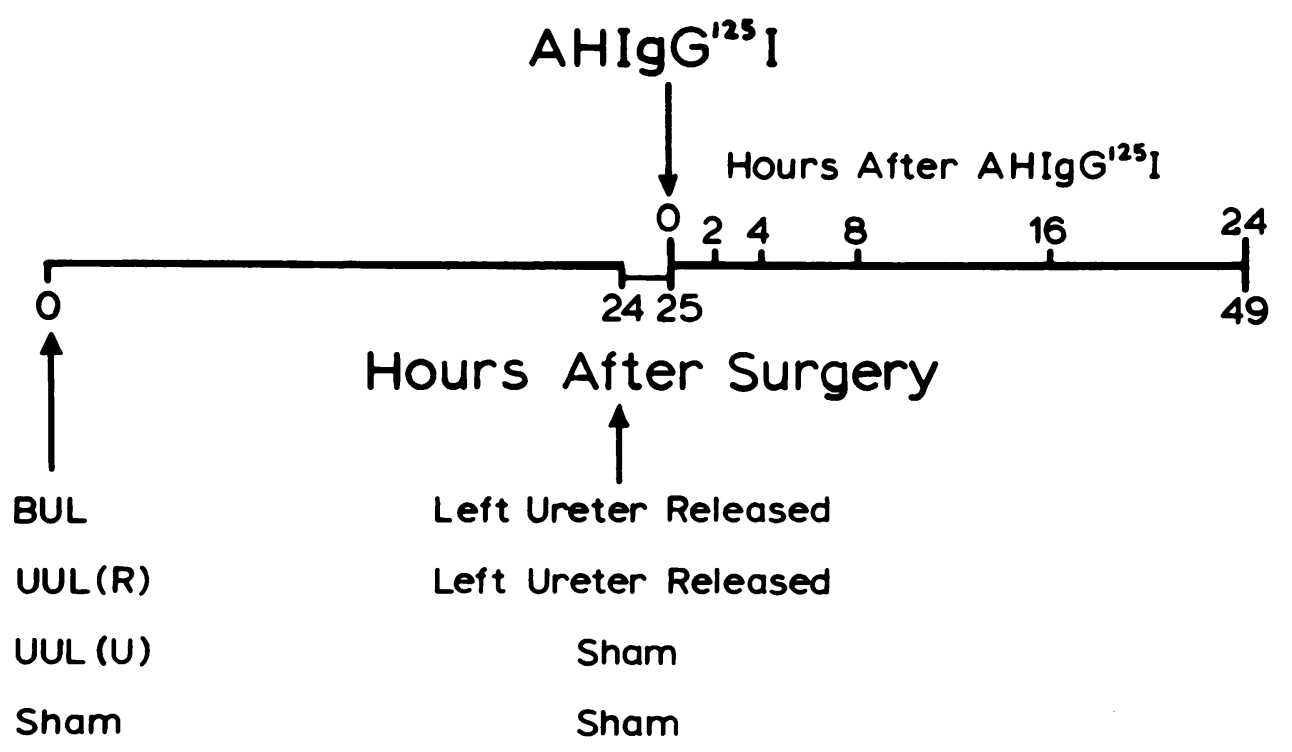

FIGURE 1 Schematic representation of the experimental design. 
groups, 25 sham-operated rats served as controls. A total of two separate experiments were performed. Physiological studies were carried out in six control animals and in groups of six animals with BUL, UUL(R), and UUL(U), $24 \mathrm{~h}$ after ureteral occlusion.

Surgical procedure. Ureteral ligation, bilateral or unilateral, was performed under light ether anesthesia. The ureters were carefully dissected, and a small piece of polyethylene tubing was placed along side of the ureter. The ureter and tubing were tied together with 3-0 silk suture. In this way, minimal damage was induced to the ureter by the ligature. After recovery from surgery, the animals were returned to their cages and allowed free access to water but no food. In sham-operated control animals for each of the groups, the ureters were dissected free of surrounding tissues but not ligated. Release of ureteral ligation was accomplished under light ether anesthesia. Care was taken to ensure that the released ureter was dissected clear of any adhesion.

Administration of $A H I g G^{125}$. All animals received AH$\operatorname{IgG}^{125} \mathrm{I}(45 \mathrm{mg} / 100 \mathrm{~g}$ body $\mathrm{wt})$ intravenously $24 \mathrm{~h}$ after ureteral ligation or sham operation. However, the $\mathrm{AHIgG}^{125} \mathrm{I}$ was given $1 \mathrm{~h}$ after ureteral release to BUL, UUL(R), and uremiacontrol rats. Groups of five experimental and five sham-operated control rats were sacrificed at $2,4,8,16$, and $24 \mathrm{~h}$ after the administration of $\mathrm{AHIgG}^{125} \mathrm{I}$.

Tissue and blood analysis. The control and experimental animals to be killed $24 \mathrm{~h}$ after the administration of AHIgG $^{125}$ I were placed in metabolic cages, and all urines were collected. Protein-bound ${ }^{125} \mathrm{I}$ in urine was investigated by the addition of $2 \mathrm{ml}$ of $10 \%$ TCA to $2 \mathrm{ml}$ of urine. From each of the experimental and control animals sacrificed at the various time intervals, the kidneys were removed; the left and right kidneys were pooled separately for determination of $A H I g G^{125} I$ in preparations of isolated glomeruli (see below). In addition, small wedge sections were taken from left and right kidneys of all control and experimental animals for histologic studies. Liver and spleen from experimental and control animals were also removed for determination of $\mathrm{AHIgG}^{125}$. The blood obtained at the time of sacrifice was used for determination of blood urea nitrogen (BUN), protein bound ${ }^{125} \mathrm{I}$, and for sucrose density gradient centrifugation.

\section{Renal histology}

Wedge-shaped renal tissue obtained at the time of sacrifice was prepared for light and immunofluorescence microscopic studies by techniques previously described (20). The intensity of immunofluorescence was arbitrarily graded from negative to $3+$. Within each specimen the intensity and pattern of fluorescence in superficial and juxtamedullary glomeruli were compared.

\section{Quantitation of AHIgG ${ }^{125}$ I in preparations of isolated glomeruli}

Glomerular isolates were prepared separately from pools of five left and five right kidneys from experimental and control animals sacrificed at the different time intervals after administration of AHIgG ${ }^{125}$ I. The kidneys were decapsulated and medulla was separated from cortex. The mash of decapsulated cortex was pushed through a 150 -mesh screen (Michigan Dynamics, Div. of Ambac Industries, Inc., Garden City, Mich.). The resulting suspension was poured through a 100mesh screen to remove large tissue fragments; the glomeruli were then collected on 200- and 325-mesh screens successively, and washed vigorously with $0.15 \mathrm{M}$ sodium chloride into a 50-ml centrifuge tube. Glomerular preparations were examined by phase microscopy to insure at least an $85 \%$ purity.
The glomeruli were then washed, lyophilized, and weighed and counted in a Biogamma II counter (Beckman Instruments, Inc., Fullerton, Calif.) for ${ }^{125} \mathrm{I}$. The data were expressed as micrograms of AHIgG ${ }^{125}$ I per milligram of dried glomeruli. Protein-bound ${ }^{125} \mathrm{I}$ in combined supernates from glomerular washes was $<0.16 \%$ of the measured radioactivity in a given pool of glomeruli, which indicates that mesangial-trapped AHIgG $^{125}$ I was not released during the washing procedure.

The contamination of isolated glomeruli by blood was demonstrated to be minimal $(0.002 \mathrm{ml}$ serum $/ \mathrm{mg}$ glomeruli) in separate experiments after administration of nonaggregated $7 \mathrm{~S}$ IgG $^{125}$; correction of glomerular concentration of AHIgG ${ }^{125}$ I for entrapped serum resulted in minimal differences from uncorrected values $(<7.0 \%){ }^{2}$

\section{Quantitation of AHIgG ${ }^{125}$ in preparations of liver and spleen}

Liver and spleen were removed from each animal at the time of sacrifice. The tissues were thoroughly washed in isotonic saline and dried in a ventilated oven at $85^{\circ} \mathrm{C}$ for $24 \mathrm{~h}$. The dried organs were weighed, placed in $1.0 \mathrm{~N}$ sodium hydroxide that contained $0.2 \mathrm{~N}$ sodium deoxycholate, and then dissolved by heating at $95^{\circ} \mathrm{C}$ for $1 \mathrm{~h}$. The dissolved tissue was then counted for ${ }^{125} \mathrm{I}$; the data were expressed as micrograms of AHIgG ${ }^{125}$ I per milligram of dried tissue. No attempt was made to correct for residual blood contamination in liver and spleen because the $\mathrm{AHIgG}^{125}$ I content in these organs was used to exclude major differences in reticuloendothelial function among experimental groups. We have not demonstrated differences in these data after perfusion of the liver with saline until free of blood. ${ }^{2}$

\section{Quantitation of $\mathrm{AHIgG}^{125}$ in blood}

Whole blood $(0.1 \mathrm{ml})$ was added to $2.0 \mathrm{ml}$ of $0.1 \%$ sodium carbonate to produce hemolysis. After total counts for ${ }^{125}$ I were obtained, $2.0 \mathrm{ml}$ of $10 \%$ TCA was added to produce protein precipitation. The samples were centrifuged at $5,000 \mathrm{~g}$, and the supernate was separated and counted to determine free ${ }^{125} \mathrm{I}$; protein bound counts were calculated by subtraction.

Serum $(0.2 \mathrm{ml})$ was layered on a $12-\mathrm{ml}$ linear sucrose gradient (10-50\% sucrose). Gradients were centrifuged at $36,000 \mathrm{rpm}$ for $16 \mathrm{~h}$ at $4^{\circ} \mathrm{C}$ with a SW 40 rotor in a Beckman ultracentrifuge (model L5-65, Beckman Instruments, Inc., Spinco Div., Palo Alto, Calif.). Fractions $(0.5 \mathrm{ml})$ were collected through a hole in the bottom of the tube and counted for ${ }^{125} \mathrm{I}$. The gradient curves were integrated, and blood levels of $\mathrm{AHIgG}^{125} \mathrm{I}$ $>7 \mathrm{~S}$ were calculated $(21)$.

\section{Determination of RBF and arterial pressure}

RBF was determined in six animals in each of the following groups: (a) control animals: RBF was determined in the left kidney $24 \mathrm{~h}$ after the sham operation; (b) BUL animals: $1 \mathrm{~h}$ after release of the left ureter, RBF was measured in both kidneys; (c) UUL(R) animals: RBF was separately determined in both kidneys $1 \mathrm{~h}$ after the release of the left ureter; and (d) UUL(U) animals: RBF was measured in both kidneys.

Animals were anesthesized with sodium pentobarbitol (50 $\mathrm{mg} / \mathrm{kg}$ of body wt) intraperitoneally, and the femoral artery was cannulated with PE 50 polyethylene tubing. Arterial pressure was monitored with a Statham P23 D6 pressure transducer (Statham Instruments Inc., Oxnard, Calif.) connected to a Hewlett-Packard recorder (model 7702b, HewlettPackard Co., Palo Alto, Calif.). RBF was measured by a small-

${ }^{2}$ Keane, W. F., and L. Raij. Unpublished observations. 
diameter flow transducer (model EP 401.5 or EP 401.8) connected to a square-wave electromagnetic flow meter (model 501, Carolina Medical Electronics, Inc., King, N. C.) and a Hewlett-Packard recorder. RBF was expressed as milliliters per minute and also as milliliters per minute per $100 \mathrm{~g}$ body weight.

\section{Micropuncture studies}

$24 \mathrm{~h}$ after either unilateral or bilateral ureteral obstruction, animals were prepared for micropuncture studies as previously described (22). Isotonic saline that contained $20 \mathrm{mg} / \mathrm{ml} \mathrm{of}$ inulin was infused via a jugular vein catheter (PE 50) at a rate of $2 \mathrm{ml} / \mathrm{h}$. Blood pressure was monitored by a second catheter placed in the left carotid artery. The ureter was released and then cannulated with PE 50 tubing. Clearance periods and micropuncture measurements were started $45 \mathrm{~min}$ after ureteral cannulation and were completed within 30-50 min. Two 15- to 20-min clearance periods were obtained. Blood was withdrawn at the midpoint of the urine collection for inulin determination.

In each rat, tubular collections and stop-flow measurements were obtained from four to eight tubules. From randomized tubular puncture sites, single nephron filtration rate was calculated from the tubular flow rate and the tubular:plasma ratio of inulin. With a servo-nulling device, the hydrostatic pressure was measured with micropipettes (OD:3-5 microns) in the proximal tubules, in the first surface convolution distal to Bowman's space. Pressures were measured under free-flow conditions and after blockage of the tubular lumen with Sudan black-stained paraffin oil. At the end of the period, $1 \mathrm{ml}$ of arterial blood was withdrawn for measurement of the systemic protein concentration. $\mathrm{P}_{\mathrm{GC}}$ was estimated with the stop-flow technique of Gertz as modified by Allison et al. (23). Estimation of $\mathrm{P}_{\mathrm{GC}}$ by stop-flow technique in normal rats has been shown to be equivalent to that measured by direct capillary puncture $(24,25)$. Tubular fluid volume was measured with a previously calibrated micropipette. Inulin concentrations in blood, tubular fluid, and urine were determined in duplicate by the microfluorometric method (22). In UUL(U) rats, no micropuncutre studies were performed. Inulin clearance was determined in the right unobstructed kidney $24 \mathrm{~h}$ after the ligation of the left kidney.

\section{Statistical analysis}

Where applicable, data are expressed as mean \pm standard error of the mean. Physiologic data were compared in control and experimental animals with the Student's $t$ test. Linear regression in the experimental and control animals was performed, and slopes were compared by standard techniques.

\section{RESULTS}

Blood levels of $>7 \mathrm{~S}$ AHIgG ${ }^{125}$ I were determined in control and experimental animals (Fig. 2). No significant differences were observed among the groups at any time interval. By $24 \mathrm{~h}$, circulating levels of $>7 \mathrm{~S}$ AHIgG${ }^{125}$ I were $<1 \%$ of the 2 -h value. Protein-bound ${ }^{125}$ I was not present in urine of either control or experimental animals.

\section{Kinetic studies of mesangial function}

Quantitative studies of $\mathrm{AHIgG}^{125}$ I in glomerular isolates. In control animals sacrificed at different time

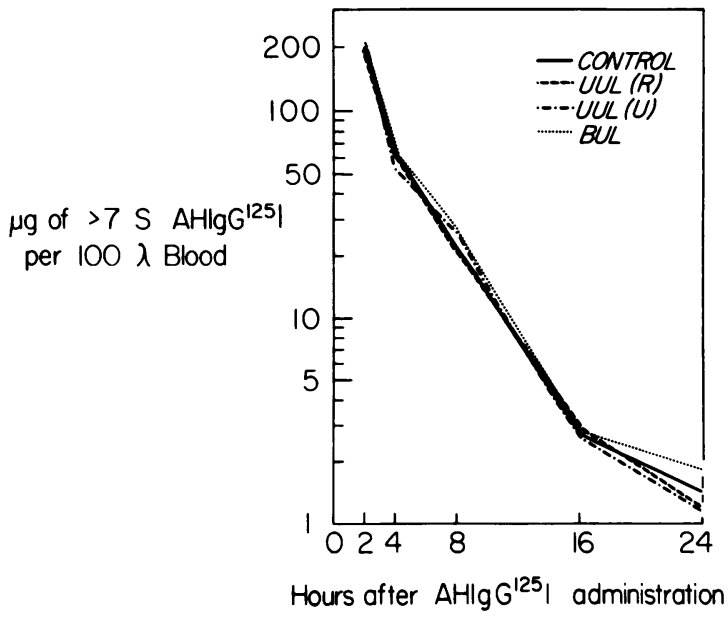

FIGURE 2 The concentrations of $>7 \mathrm{~S} \mathrm{AHIgG}^{125} \mathrm{I}$ in blood of control and experimental animals determined at various time intervals. Each plot represents the mean value of data obtained from two separate experiments.

intervals, the concentration of glomerular $\mathrm{AHIgG}^{125} \mathrm{I}$ was highest at $2 \mathrm{~h}$, which was the first time period evaluated (Figs. 3 and 4). Thereafter, a progressive decrease in concentration was observed that was linear when expressed as the logarithm of glomerular AHIgG${ }^{125}$ I concentration vs. time after injection. By $4 \mathrm{~h}$, glomerular AHIgG ${ }^{125}$ I had decreased to $68 \%$ of the 2 -h value and by $24 \mathrm{~h}$ to $14 \%$. Similar plots of glomerular AHIgG${ }^{125}$ I vs. time were found on analysis of glomeruli from kidneys of sham-operated control animals and nonob-

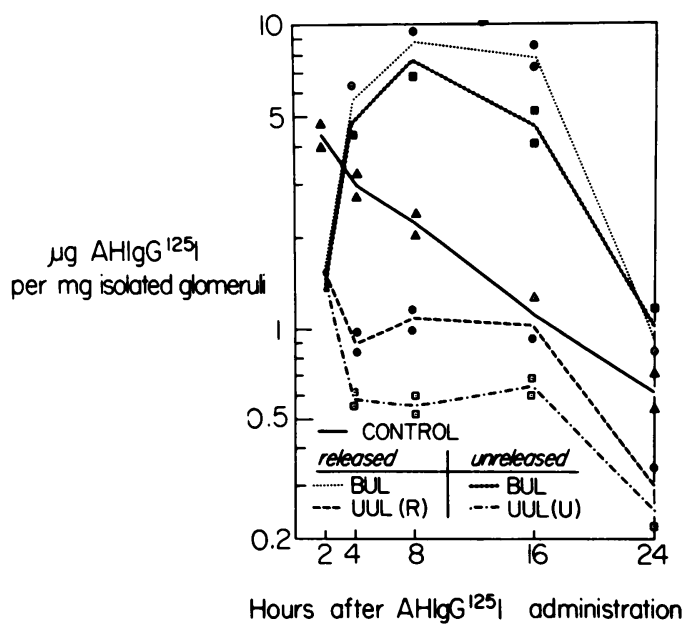

FIGURE 3 The concentration of $\mathrm{AHIgG}^{125} \mathrm{I}$ in glomeruli isolated from control kidneys and from kidneys with ureteral obstruction. Each plot represents the mean value of data obtained from two separate experiments. Each point represents the concentration of glomeruli from five kidneys in each group at the time intervals noted. Data points at $2 \mathrm{~h}$ are not represented to avoid crowding; variations between experiments were similar to that observed at other time intervals. 


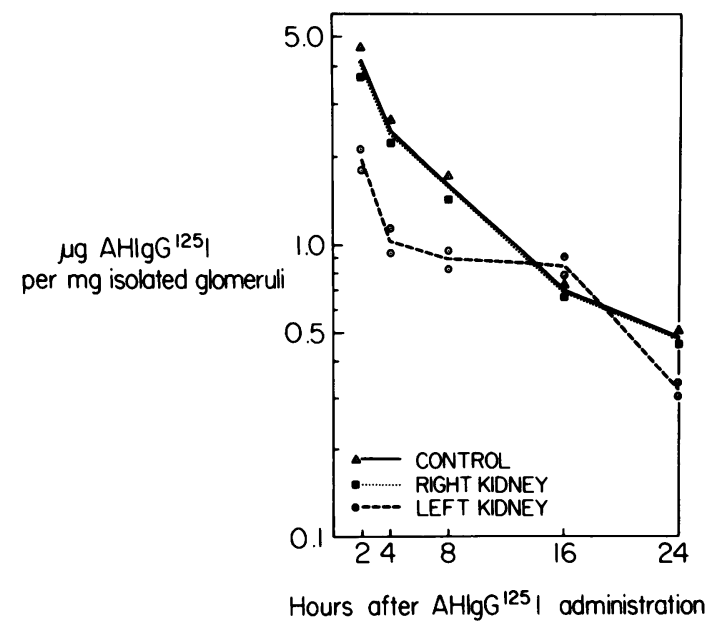

FIGURE 4 Mesangial concentrations of AHIgG ${ }^{125}$ I in glomeruli isolated from control animals and from animals with one ureter ligated for $24 \mathrm{~h}$ and then released (left kidney) and the other ureter severed and allowed to drain into the peritoneal cavity (right kidney). The concentration of AHIgG${ }^{125}$ I in blood, spleen, and liver (not shown) were not different from values obtained in BUL, UUL(R), and UUL(U) animals.

structed kidneys of $\mathrm{UUL}(\mathrm{R})$ and $\mathrm{UUL}(\mathrm{U})$ animals. At $2 \mathrm{~h}$, glomerular AHIgG ${ }^{125}$ in $\mathrm{UUL}(\mathrm{R}), \mathrm{UUL}(\mathrm{U})$, and both BUL kidneys, was $\cong 30 \%$ of the levels observed in control animals (Fig. 3). Between 2 and $4 \mathrm{~h}$, the concentration increased in both BUL kidneys but decreased further in $\mathrm{UUL}(\mathrm{U})$ and in $\mathrm{UUL}(\mathrm{R})$ kidneys.

In all obstructed kidneys, glomerular concentrations of $\mathrm{AHIgG}^{125}$ I remained relatively constant between 4 and $16 \mathrm{~h}$. However, in BUL (released or unreleased), glomerular concentration of aggregates from 4 to $16 \mathrm{~h}$ was $\cong 10$-fold those in UUL(U) and UUL(R) kidneys. In all experimental groups, after $16 \mathrm{~h}$, at a time when the blood levels of $\mathrm{AHIgG}^{125}$ I had decreased to $<3 \%$ of initial values, there was a marked decrease in mesangial AHIgG ${ }^{125}$.

The BUN was elevated in animals with BUL and in the uremia-control rats $184.67 \pm 10.61$ and 155.0 $\pm 11.3 \mathrm{mg} / \mathrm{dl}$, respectively. Mesangial kinetics in the latter group were similar to that observed in UUL(R) rats (Fig. 4), which demonstrated that uremia per se did not influence mesangial kinetics of $\mathrm{AHIgG}^{125} \mathrm{I}$.

\section{Immunofluorescence and light microscopy}

At $2 \mathrm{~h}$, human AHIgG, detected with fluorescein isothiocyanate-labeled goat anti-human IgG, was present primarily within the mesangium but also in a subendothelial distribution. By $4 \mathrm{~h}$, human IgG was found almost entirely within the mesangium. Minimal fluorescence was seen in peritubular capillaries, but none was observed in tubules or Bowman's space. This pattern of fluorescence was similar in control and experimental kidneys. However, the intensity of fluorescent staining was different in the control and experimental kidneys at the various time intervals but showed an excellent correlation with the quantitative determination of $\mathrm{AHIgG}^{125} \mathrm{I}$ in isolated glomeruli. In control and nonobstructed kidneys of $U U L(U)$ and $U U L(R)$, mesangial immunofluorescence was intense $(2-3+)$ at 2,4 , and $8 \mathrm{~h}$ after administration of $\mathrm{AHIgG}^{125} \mathrm{I}$, with a decrease to trace amounts by $24 \mathrm{~h}$. In obstructed kidneys from $U U L(R)$ and $U U L(U)$, the intensity of immunofluorescence $(1+)$ was the same at all time periods and became negative by $24 \mathrm{~h}$. Evaluation of BUL kidneys, whether released or unreleased, revealed striking fluorescence $(3+)$ at 4,8 , and $16 \mathrm{~h}$, with a lesser intensity $(1+)$ at 2 and $24 \mathrm{~h}$. In addition, tissue obtained by wedge biopsy from control and experimental animals revealed that at all time intervals studied there were no differences in the intensity of mesangial staining between cortical and deep glomeruli. Light microscopy revealed only tubular dilatation, mild epithelial cell flattening, and interstitial edema in obstructed kidneys.

\section{AHIgG ${ }^{125}$ I in liver and spleen}

Despite striking differences in mesangial $\mathrm{AHIgG}^{125} \mathrm{I}$ between control and experimental animals, there were no major differences in $\mathrm{AHIgG}^{125}$ I concentrations in the liver and spleens among control and $U U L(U)$, UUL(R), and BUL rats (Fig. 5). In both organs there was a progressive decrease in $\mathrm{AHIgG}^{125}$ I concentration, and, by $24 \mathrm{~h},<1 \%$ of the initial concentration was present. Linear regression analysis of the slopes derived from data for liver and spleen showed no significant differences between experimental and control animals.

\section{Physiological studies}

RBF was significantly decreased in all models of ureteral obstruction (Table I). The RBF of unreleased kidneys in BUL and $U U L(U)$ animals was reduced to

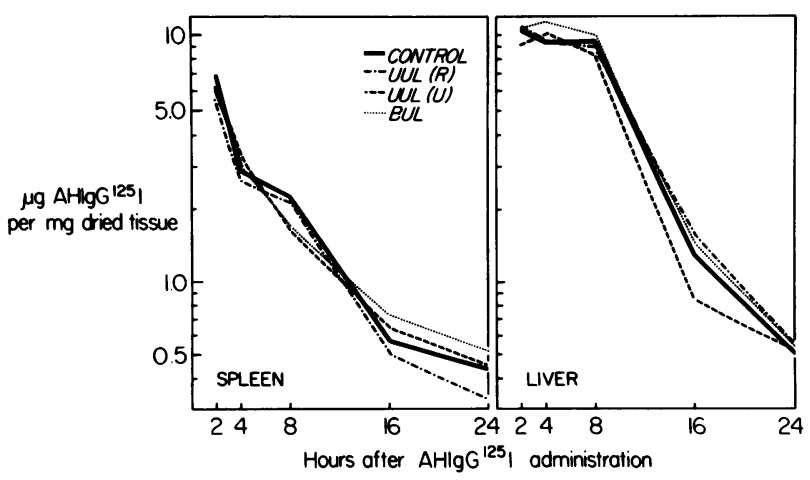

Figure 5 The concentration of $\mathrm{AHIgG}^{125} \mathrm{I}$ in livers and spleens of control and experimental animals sacrificed at various time intervals. Each plot represents the mean value of data obtained from two separate experiments. 
TABLE I

Renal Blood Flow in Control and Experimental Animals*

\begin{tabular}{|c|c|c|c|c|c|c|c|}
\hline & \multirow{2}{*}{$\frac{\text { Control }}{\text { LK }}$} & \multicolumn{2}{|c|}{ BUL } & \multicolumn{2}{|c|}{ UUL(R) } & \multicolumn{2}{|c|}{ UUL(U) } \\
\hline & & $\operatorname{LK}(\mathrm{R})$ & $\mathrm{RK}(\mathrm{U})$ & $\operatorname{LK}(\mathrm{R})$ & $\mathrm{RK}(\mathrm{N})$ & $\mathrm{LK}(\mathrm{U})$ & $\mathrm{RK}(\mathrm{N})$ \\
\hline $\mathrm{ml} / \mathrm{min}$ & $5.90 \pm 0.18$ & $5.07 \pm 0.19 \ddagger \S$ & $4.16 \pm 0.20^{\| 1}$ & $4.60 \pm 0.23 \ddagger$ & $6.22 \pm 0.13^{* *}$ & $3.54 \pm 0.30^{\prime \prime}$ & $5.85 \pm 0.23^{* *}$ \\
\hline $\mathrm{ml} / \mathrm{min} / 100 \mathrm{~g}$ body $w t$ & $2.84 \pm 0.03$ & $2.44 \pm 0.11 \ddagger \S$ & $1.73 \pm 0.11^{\| \rrbracket}$ & $2.03 \pm 0.08 \ddagger$ & $3.08 \pm 0.11^{* *}$ & $1.47 \pm 0.06^{\prime \prime}$ & $3.02 \pm 0.04^{* *}$ \\
\hline
\end{tabular}

LK, left kidney; RK, right kidney; (R), released; (U), unreleased; (N), normal.

$* n=6$.

$\$ P<0.05$ compared with control.

$\S P<0.05$ compared with LK of UUL(R).

" $P<0.001$ compared with control.

I Not significantly different from LK of UUL(U).

** Not significantly different from control.

$50 \%$ of control kidneys. In released kidneys of BUL and $\mathrm{UUL}(\mathrm{R})$ animals RBF was $86 \%$ and $71 \%$ of control kidneys, respectively. Compared with control as well as $\operatorname{UUL}(\mathrm{R})$ and $\mathrm{UUL}(\mathrm{U})$, BUL animals had a higher arterial pressure and BUN (Table II).

Comparison of glomerular dynamic factors between released kidneys of BUL and $\mathrm{UUL}(\mathrm{R})$ animals is presented in Table II. After ureteral release, the hydrostatic pressures in the proximal tubules were lower than normal in both models. Compared with control rats, single nephron glomerular filtration rate (SNGFR) and the calculated $\mathrm{P}_{\mathrm{GC}}$ were reduced in the released kidneys of BUL and UUL animals. It is apparent that in both of these models the reduced $\mathrm{P}_{\mathrm{GC}}$ can be explained by vasoconstriction of the afferent arteriole. Calculations of resistance changes across the afferent arteriole from the changes in blood flow and pressures indicated that the increase in resistance was similar in both $\mathrm{UUL}(\mathrm{R})$ and BUL kidneys. However, $\mathrm{P}_{\mathrm{GC}}$ in released kidneys of BUL animals was higher than in the released kidneys of UUL(R) animals. This increase can be explained by the higher mean arterial pressure observed in BUL animals (Table II). However, SNGFR was not significantly different between BUL and UUL(R) kidneys. This suggests that the glomerular capillary ultrafiltration coefficient, may be lower in BUL than UUL(R) kidneys. Whole kidney glomerular filtration rate (GFR) in both models was proportionately more decreased than SNGFR, which suggests nephron GFR heterogeneity, although back leak of glomerular filtrate (13) or sample errors may be contributory factors. Although these variables conceivably could affect the accuracy of the data, the methods and results are similar to that reported by other investigators $(9,10,12)$.

\section{DISCUSSION}

After the administration of $\mathrm{AHIgG}^{125}$ I to the normal rat, kinetic studies have shown a rapid increase in

TABLE II

Micropuncture Data, and BUN in Control and Experimental Animals

\begin{tabular}{|c|c|c|c|c|c|c|c|c|c|}
\hline & UV & $\mathrm{C}_{\text {In }}$ & A & SNGFR & $P_{t}$ & SFP & $\mathrm{P}_{\mathrm{GC}}$ & MAP & BUN \\
\hline & $\mu l / \min$ & $\mathrm{ml} / \mathrm{min}$ & $m m H g$ & $n l / m i n$ & $m m H g$ & $m m H g$ & $m m H g$ & $m m H g$ & $m g / d l$ \\
\hline \multicolumn{10}{|l|}{ Control $(n=6)$} \\
\hline Mean & 3.96 & 0.90 & 14.10 & 26.05 & 13.10 & 39.75 & 53.82 & 126.5 & 25.52 \\
\hline SEM & \pm 1.55 & \pm 0.19 & \pm 1.10 & \pm 2.05 & \pm 0.41 & \pm 0.25 & \pm 0.15 & \pm 1.5 & \pm 1.04 \\
\hline \multicolumn{10}{|l|}{ BUL $(n=6)$} \\
\hline Mean & 29.13 & 0.18 & 13.41 & 10.43 & 10.62 & 25.91 & 39.33 & 147.4 & 184.67 \\
\hline SEM & \pm 5.06 & \pm 0.03 & \pm 0.79 & \pm 1.25 & \pm 0.81 & \pm 1.57 & \pm 1.82 & \pm 1.8 & \pm 10.61 \\
\hline \multicolumn{10}{|l|}{$\operatorname{UUL}(\mathrm{R})(n=6)$} \\
\hline Mean & 1.36 & 0.14 & 13.82 & 9.84 & 8.85 & 16.68 & 30.50 & 119.4 & 23.69 \\
\hline SEM & \pm 0.81 & \pm 0.02 & \pm 0.22 & \pm 0.96 & \pm 1.55 & \pm 0.84 & \pm 1.50 & \pm 1.2 & \pm 1.21 \\
\hline \multicolumn{10}{|l|}{$P$ value } \\
\hline Control vs. BUL & $<0.01$ & $<0.001$ & NS & $<0.001$ & NS & $<0.01$ & $<0.001$ & $<0.001$ & $<0.001$ \\
\hline Control vs. UUL(R) & $<0.05$ & $<0.001$ & NS & $<0.001$ & $<0.05$ & $<0.05$ & $<0.001$ & NS & NS \\
\hline BUL vs. UUL(R) & $<0.005$ & NS & NS & NS & NS & $<0.05$ & $<0.01$ & $<0.001$ & $<0.001$ \\
\hline
\end{tabular}

$\mathrm{UV}$, urine volume; $\mathrm{C}_{\mathrm{In}}$, inulin clearance; $\mathrm{A}$, plasma oncotic pressure; $\mathrm{P}_{\mathrm{t}}$, tubular pressure; $\mathrm{SFP}$, stop-flow pressure; MAP, mean arterial pressure. 
mesangial uptake within a period of 2-4 $\mathrm{h}$ after administration, followed by a decline over the subsequent $24 \mathrm{~h}$. The initial uptake, which occurs during a period of relatively high blood levels, and the subsequent falloff can be described in terms of afferent and efferent limbs, respectively (8). These phases are not separate events because efferent mechanisms likely come into play during the initial uptake period, and, therefore, no time line can clearly separate one phase from the other.

Prior studies have demonstrated that uptake of AHIgG, immune complexes, and other macromolecules may be increased $(a)$ after the elevation of the blood level, either by raising the dose or by impairing systemic mononuclear phagocytic uptake $(5,7,26,27)$, and (b) after the administration of anti-GBM antibody or aminonucleoside of puromycin $(6,7)$. In both situations the efferent limb appears to be intact. The localization of AHIgG in the mesangium is dependent upon delivery rate to the capillary lumen (the concentration in the blood times glomerular blood flow) as well as on transfer from the lumen to the mesangium. In all models of ureteral obstruction reported here, we observed a marked decrease in 2-h mesangial AHIgG ${ }^{125}$ I. Because at the time, blood levels of $\mathrm{AHIgG}^{125}$ I in experimental and control animals were high, this reduced uptake suggests a decrease in either delivery of $\mathrm{AHIgG}^{125} \mathrm{I}$ or in transfer from the capillary to the mesangium (lumenmesangial transfer) or in both. Indeed, a decrease in delivery rate of aggregates produced by a $50 \%$ reduction in $\mathrm{RBF}$ secondary to aortic constriction causes a proportionate decrease of the same magnitude in the mesangial $\mathrm{AHIgG}^{125} \mathrm{I}^{2}{ }^{2}$ The reduction in RBF to $50 \%$ of normal in $U U L(U)$ and in unreleased BUL kidneys could be the reason for diminished 2-h uptake of $\mathrm{AHIgG}^{125} \mathrm{I}$ in these models. However, this does not explain completely the similar decrease in 2-h mesangial $\mathrm{AHIgG}^{125} \mathrm{I}$ in released BUL and UUL(R) kidneys, which have a higher $\mathrm{RBF}, 86 \%$ and $71 \%$ of normal, respectively. Hence, a similar decrease in 2-h mesangial $\mathrm{AHIgG}^{125}$ in all experimental models despite different RBF would suggest that impaired lumen-mesangial transfer of aggregates may also be a contributing factor. If this assumption is correct, lumen-mesangial transfer of aggregates in BUL kidneys is less impaired than in UUL kidneys.

Mesangial AHIgG ${ }^{125}$ in BUL kidneys increased in the period from 2-4 h, whereas in $U U L(R)$ and $U U L(U)$, mesangial $\mathrm{AHIgG}^{125}$ I decreased. The reason for this difference is unknown, although several explanations may be entertained. First, this may be a consequence of differences in capillary pressure and/or permeability. The capillary pressure was lower than normal in all models of ureteral obstruction of 24-h duration but was higher in BUL and UUL(R) kidneys. In addition, although not specifically determined in these experiments, the glomerular capillary ultrafiltration coefficient appeared lower in BUL than UUL(R). Second, the higher concentration of $\mathrm{AHIgG}^{125} \mathrm{I}$ at $4 \mathrm{~h}$ in BUL compared with UUL may result from the differences in uptake described above superimposed on efferent mesangial blockade (see below). Third, these findings may be related to differences in the time-course of uptake, i.e., rapid in UUL so that maximal uptake had already occurred by $2 \mathrm{~h}$, and retarded in BUL with maximum uptake at 4-8 h. However, this latter hypothesis does not explain the plateau at $4-16 \mathrm{~h}$ in UUL animals.

Ureteral release did not substantially alter mesangial kinetics in either BUL or UUL kidneys, which suggests that the factors inducing abnormal kinetics persisted after release of ureteral obstruction. Other studies have shown that alterations in glomerular function persist for at least $24 \mathrm{~h}$ after release of ureteral obstruction (28).

The mechanisms that control the disappearance of macromolecules from the mesangium or efferent limb are unclear. In previous studies, intracellular degradation by mesangial cells was postulated as a primary mechanism $(1,29)$. However, the predominant localization of certain macromolecules such as antigen-antibody complexes (30) and aggregated proteins (5) within the mesangial channels and not within mesangial cells suggests that this may not be the main mechanism. Passage by way of the glomerular stalk to the JG zone has been clearly demonstrated with morphologic techniques, although the pathway thereafter is obscure $(31,32)$. It is possible that drainage into the lymphatics, interstitium, or tubules may occur, although none of these routes has been proven. Regurgitation into the glomerular circulation is theoretically possible but has not been shown by morphologic techiques. For any given blood level of macromolecules, mesangial concentration is determined by a dynamic process that depends upon the balance between the afferent and efferent limb. As previously noted, after intravenous injection into normal animals, blood and glomerular levels of $\mathrm{AHIgG}^{125}$ I decreased linearly over $24 \mathrm{~h}$. The disappearance of macromolecules in obstructed kidneys from $\mathrm{UUL}(\mathrm{R}), \mathrm{UUL}(\mathrm{U})$, and BUL animals was markedly different from normal: a common feature in all three groups was the lack of a significant decrease in mesangial AHIgG $^{125}$ I between 4 and $16 \mathrm{~h}$ despite the progressive decrease in blood levels of $\mathrm{AHIgG}^{125}$ I. These findings suggest a blockade of the mesangial efferent limb. The level of mesangial $\mathrm{AHIgG}^{125} \mathrm{I}$ at which the 4 to 16 -h plateau occurred was different among the experimental models, and, in each model, a function of the mesangial concentration of $\mathrm{AHIgG}^{125} \mathrm{I}$ present at $4 \mathrm{~h}$. In that respect, in both the released and unreleased kidneys of BUL animals, this plateau was 10 -fold greater than that 
observed in UUL(U) or UUL(R). The observed alterations in mesangial kinetics were a result of intrarenal factors because there were no differences in the blood levels of AHIgG ${ }^{125}$ I among any of the groups. During the 16- to 24-h period of time, a progressive decrease in mesangial $\mathrm{AHIgG}^{125}$ I occurred in obstructed as well as control kidneys.

The reason for the blockade in the transport of $\mathrm{AHIgG}^{125}$ I out of the mesangium of kidneys with ureteral obstruction is unclear. During the 4- to $16-\mathrm{h}$ time period, the concentration of $\mathrm{AHIgG}^{125} \mathrm{I}$ in the blood was relatively high, which suggests that the lack of decrease in mesangial concentration was dependent upon high levels of circulating AHIgG ${ }^{125}$ I. However, during the 16- to 24-h time period, a significant decrease in mesangial concentration occurred in all models associated with blood levels that were $<3 \%$ of initial values. These observations suggest at least two mechanisms for disposal of macromolecules from the mesangium: one which is abrogated by ureteral obstruction and is detected at high levels of circulating AHIgG ${ }^{125}$ I and the other which is not affected by ureteral occlusion and is evident at low blood concentrations. One possibility to explain these observations is that ureteral obstruction impairs traffic by way of the glomerular stalk to the JG region. At high blood levels, glomerular concentrations change little because of a dynamic equilibrium between the circulating and mesangial pools of AHIgG ${ }^{125}$ I. As the blood concentration decreases to low values at the 16- to 24-h time period, this equilibrium becomes imbalanced, and, despite continued ureteral obstruction, egress from the mesangium occurs. This loss may be a consequence of regurgitation of $\mathrm{AHIgG}^{125} \mathrm{I}$ into the circulation by way of the glomerular capillary and/or degradation after pinocytosis and phagocytosis.

Whereas previous physiologic and morphologic studies in animals with ureteral obstruction suggest the nephron function may be heterogenous (10), we were unable to demonstrate differences between cortical and juxtamedullary glomerular uptake of $\mathrm{AHIgG}^{125} \mathrm{I}$ in any of the models using semiquantitative immunofluorescence microscopy. However, with this technique, subtle differences in mesangial AHIgG ${ }^{125}$ I may not be appreciated. From the studies performed in uremia-control rats, it is apparent that the differences in the mesangial kinetics between BUL, UUL, and control animals cannot be explained by uremia per se. In addition, in other studies we have shown that rats made acutely uremic with mercury chloride have normal mesangial uptake and release of $\mathrm{AHIgG}^{125} \mathrm{I}$ (33).

The analysis of physiologic parameters measured in these experiments suggests that no single factor can explain the observed differences in mesangial kinetics. These studies do, however, demonstrate that the altera- tions induced by ureteral obstruction markedly influence the afferent and efferent limb of the mesangium. The possibility that some of these changes in mesangial kinetics may be related to retention or increased production of vasoactive materials cannot be excluded. Experimental data have shown that hydronephrotic kidneys produce increased amounts of the vasodilator prostaglandin $\mathrm{E}_{2}$ and the vasoconstrictor thromboxane $A_{2}(34-36)$. It is interesting to speculate that these mediators or similar vasoactive substances, including angiotensin II (37), could modulate the mesangial afferent and/or efferent limbs by acting upon the contractile elements of the mesangium.

Clinically, the experiments reported here may have a bearing upon the recent report of Mathew et al. (38), who demonstrated that transplanted kidneys with persistent ureteral reflux develop mesangial proliferative changes, findings not present in the original kidneys.

\section{ACKNOWLEDGMENTS}

We are grateful to Dr. Franklyn G. Knox, Department of Physiology, Mayo Clinic, Rochester, Minn., in whose laboratory Dr. Osswald performed the micropuncture experiments. We acknowledge the technical assistance of Nan Neus, Barbara Wrigley, and Diane Dancil, and the graphic assistance of Joan Mack. Cindy Tollefson and Bridget Stellmacher typed the manuscript.

This investigation was supported in part by grants from the Minnesota Medical Foundation and the Graduate School of the University of Minnesota as well as National Institutes of Health grants AI 10704, HL 06314, and IROIAM2251801.

\section{REFERENCES}

1. Farquhar, M. G., and G. E. Palade. 1962. Functional evidence for the existence of a third cell type in the renal glomerulus. J. Cell Biol. 13: 55-87.

2. Barajas, L. 1970. The ultrastructure of the juxtaglomerular apparatus as disclosed by three-dimensional reconstructions from serial sections. J. Ultrastruct. Res. 33: 116-147.

3. Becker, C. G. 1972. Demonstration of actomyosin in mesangial cells of the renal glomerulus. Am. J. Pathol. 66: 97-106.

4. Scheinman, J. I., A. J. Fish, and A. F. Michael. 1974. The immunohistopathology of glomerular antigens. The glomerular basement membrane, collagen, and actomyosin antigens in normal and diseased kidneys. J. Clin. Invest. 54: $1144-1154$.

5. Michael, A. F., A. J. Fish, and R. A. Good. 1967. Glomerular localization and transport of aggregated protein in mice. Lab. Invest. 17: 14-29.

6. Mauer, S. M., A. J. Fish, E. B. Blau, and A. F. Michael. 1972. The glomerular mesangium. I. Kinetic studies of macromolecular uptake in normal and nephrotic rats. $J$. Clin. Invest. 51: 1092-1101.

7. Mauer, S. M., A. J. Fish, N. K. Day, and A. F. Michael. 1974. The glomerular mesangium. II. Studies of macromolecular uptake in nephrotoxic nephritis in rats. J. Clin. Invest. 53: 431-439.

8. Michael, A. F., T. E. Nevins, L. Raij, W. F. Keane, and J. Scheinman. 1979. Macromolecular transport in the glomerulus: studies of the mesangium and epithelium 
in vivo and in vitro. In Contemporary Issues in Nephrology. B. M. Brenner and J. H. Stein, editors. Churchill Livingstone, N. Y. 3: 167-213.

9. Yarger, W. E., H. S. Aynedjian, and N. Bank. 1972. A micropuncture study of postobstructive diuresis in the rat. J. Clin. Invest. 51: 625-637.

10. Harris, R. H., and W. E. Yarger. 1974. Renal function after release of unilateral ureteral obstruction in rats. Am.J. Physiol. 227: 806-815.

11. Harris, R. H., and W. E. Yarger. 1975. The pathogenesis of post-obstructive diuresis. The role of circulating natriuretic and diuretic factors including urea.J. Clin. Invest. 56: $880-887$.

12. Jaenike, J. R. 1972. The renal functional defect of postobstructive nephropathy. The effects of bilateral ureteral obstruction in the rat. J. Clin. Invest. 51: 2999-3006.

13. Wilson, D. R. 1977. Renal function during and following obstruction. Annu. Rev. Med. 28: 329-339.

14. Buerket, J., E. Alexander, M. L. Purkerson, and S. Klahr. 1976. On the site of decreased fluid reabsorption after release of ureteral obstruction in the rat. J. Lab. Clin. Med. 87: 397-410.

15. Schnermann, J., E. Perssan, H. Ulfendehl, M. Wolgast, and P. Wunderlich. 1972. Functional characteristics of the renal interstitial space. In Recent Advances in Renal Physiology. S. Karger GmbH, Munich, Germany. 43-54.

16. Vogel, G., K. Gartner, M., Ullrich. 1974. The flow rate and macromolecular content of hilar lymph from the rabbit kidney under conditions of renal venous pressure elevation and restriction of renal function. Studies of the origin of renal lymph. Lymphology. 7: 176-189.

17. McConahey, P. J., and F. J. Dixon. 1966. A method of trace iodination of protein for immunologic studies.J. Int. Arch. Allergy Appl. Immunol. 29: 185-189.

18. Christian, C. L. 1960. Studies of aggregated gamma globulin. I. Sedimentation, electrophoretic and anticomplementary properties. J. Immunol. 84: 112-116.

19. Ishizaka, K., and T. Ishizaka. 1960. Biologic activity of aggregated gamma globulin. A study of various methods for aggregation and species differences. J. Immunol. 85: $163-171$.

20. Raij, L., W. F. Keane, and A. F. Michael. 1977. Unilateral Schwartzman reaction. Cortical necrosis in one kidney following in vivo perfusion with endotoxin. Kidney Int. 12: 91-95.

21. Hinton, R., and M. Dobrota. 1976. Density gradient centrifugation. Laboratory Techniques in Biochemistry and Molecular Biology. T. S. Work and E. Work, editors. North Holland Publishing Co., Amsterdam.

22. Schnermann, J., H. Osswald, and M. Hermle. 1977. Inhibitory effect of methylxanthines on feedback control of glomerular filtration rate in the rat kidney. Pfugers Arch. Eur. J. Physiol. 369: 39-48.

23. Allison, M., E. M. Cipham, and C. W. Gottschalk. 1972. Hydrostatic pressure in the rat kidney. Am. J. Physiol. 223: 975-983.
24. Kallskog, O., L. O. Lindbom, H. R. Ulfendahl, and $M$. Wolgast. 1975. Kinetics of the glomerular ultrafiltration in the rat kidney. An experimental study. Acta Physiol. Scand. 95: 293-300.

25. Azar, S., M. A. Johnson, B. Hertel, and L. Tobian. 1977. Single-nephron pressures, flows and resistances in hypertensive kidneys with nephrosclerosis. Kidney Int. 12: 28-40.

26. Haakenstad, A. O., and M. Mannick. 1974. Saturation of the reticuloendothelial system with soluble immune complexes. J. Immunol. 112: 1939-1948.

27. Haakenstad, A. O., and M. Mannick. 1976. The disappearance kinetics of soluble immune complexes prepared with reduced and alkylated antibodies and with intact antibodies in mice. Lab. Invest. 35: 283-292.

28. McDougal, W. S., and F. S. Wright. 1972. Defect in proximal and distal sodium transport in post-obstructive diuresis. Kidney Int. 2: 304-317.

29. Vernier, R. L., S. M. Mauer, A. J. Fish, and A. F. Michael. 1971. The mesangial cell in glomerulonephritis. In Advances in Nephrology. J. Hamburger, J. Crosnier, and M. Maxwell, editors. Year Book Medical Publishers, Inc., Chicago. 1: 31-46.

30. Striker, G. E., M. Mannik, and M. Y. Tung. 1979. Role of marrow-derived monocytes and mesangial cells in removal of immune complexes from renal glomeruli. $J$. Exp. Med. 149: 127-136.

31. Leiper, J. M., D. Thomason, and M. K. MacDonald. 1977. Uptake and transport of imposil by the glomerular mesangium in the mouse. Lab. Invest. 37: 526-533.

32. Elema, J. D., J. R. Hoyer, and R. L. Vernier. 1976. The glomerular mesangium: uptake and transport of intravenously injected colloidal carbon in rats. Kidney Int. 9: 395-406.

33. Keane, W. F., S. Azar, and L. Raij. 1977. Glomerular, mesangial and tubular function in mercury chloride $\left(\mathrm{HgCl}_{2}\right)$ acute renal failure. Clin. Res. 25: 437A.

34. Nishikaw, K., A. Morrison, and P. Needleman. 1977. Exaggerated prostaglandin biosynthesis and its influence on renal resistance in the isolated hydronephrotic rabbit kidney. J. Clin. Invest. 59: 1143-1150.

35. Morrison, A. R., K. Nishikawa, and P. Needleman. 1978. Thromboxane $A_{2}$ biosynthesis in the ureter obstructed isolated perfused kidney of the rabbit. J. Pharmacol. Exp. Ther. 205: 1-8.

36. Gerber, J. G., J. L. Data, and A. S. Nies. 1978. Enhanced renal prostaglandin production in the dog. The effect of sodium arachidonate in nonfiltering kidney. Circ. Res. 42: 43-45.

37. Yarger, W. E., and R. H. Harris. 1978. Angiotensin II (AgII) a vasoconstrictor in the post-obstructed kidney (POK) of the rat. Kidney Int. 14: 735. (Abstr.)

38. Mathew, T.H., H. Smith-Kincaid, and P. Vikraman. 1977. Risks of vesicoureteric reflux in the transplanted kidney. New Engl. J. Med. 297: 414-418. 